\title{
Hard and thermal probes of QGP from the perspective of Lattice QCD
}

\author{
Heng-Tong Ding \\ Key Laboratory of Quark $\mathcal{E}$ Lepton Physics (MOE) and Institute of Particle Physics, \\ Central China Normal University, Wuhan 430079, China
}

\begin{abstract}
In this talk I review the current status of lattice QCD results on the hard and thermal probes of QGP, including jet quenching parameters, the melting of quarkonia and open heavy flavours, thermal photon/dilepton rates, electrical conductivity as well as heavy quark diffusion coefficients.
\end{abstract}

Keywords: Lattice QCD, QGP, heavy ion collisions

\section{Introduction}

The primary goal of heavy ion programs at the RHIC and LHC experiments is to explore the properties of nuclear matter under extreme conditions. It has been confirmed by experimentalists at RHIC and LHC that a new matter, i.e. Quark Gluon Plasma (QGP) is formed in the relativistic heavy ion collisions. However, properties of QGP are not yet fully understood quantitatively. This requires efforts from both the analysis of experimental data and theoretical modelling. Lattice QCD calculations, which are based on first principles, can provide crucial inputs to phenomenological studies of QGP properties. In the following I will review the current status of lattice QCD calculations on the deconfinement, electromagnetic and transport properties of QGP.

\section{Deconfinement properties of QGP}

It is now well-known that the transition of nuclear matter from hadronic phase to quark gluon plasma phase is not a true phase transition but a rapid crossover. The transition temperature $T_{c}$ of such a rapid crossover is around 154 $\mathrm{MeV}$ [1, 2]. Unlike light hadrons which probe the chiral aspect of the QCD transition, e.g. the degeneracy of vector meson $\rho$ and axial-vector meson $a_{1}$ signals the restoration of chiral symmetry, the fate of heavy hadrons in the hot and dense medium reflects more on the deconfinement properties of QGP, e.g. they may survive at temperatures above $T_{c}$ due to their small sizes and large binding energies [3].

The abundance production of strange hadrons in heavy ion collisions compared to proton-proton collisions is considered as one of the signatures that QGP is formed. This is based on the fact that there are no strange valence quarks in the initial colliding nuclei. However, it is not yet clear from the theoretical side whether strange hadrons survive above $T_{c}$ or not. One may think that since the strange quark is much heavier than the light quark and is not much affected by the chiral symmetry then the strange hadrons may survive above $T_{c}$. The understanding of the fate of strange hadrons in the hot medium also affects our thinking on its freeze-out temperature. 
One way to investigate the fate of open strange hadron in the hot medium is to check the fluctuations and correlations of conserved quantum numbers, e.g. the baryon number (B) and electric charge (Q). By comparing these quantities calculated from lattice QCD with those calculated from Hadron Resonance Gas (HRG) model at lower temperatures one can get an idea about at what temperature the new degrees of freedom start to emerge.

The diagonal cumulants and their off-diagonal correlations are defined as the derivatives of pressure with respect to various chemical potentials $\hat{\mu}_{X}=\mu_{X} / T$

$$
\chi_{\mathrm{mn}}^{\mathrm{XY}}=\left.\frac{\partial^{(m+n)}\left(p\left(\hat{\mu}_{X}, \hat{\mu}_{Y}\right) / T^{4}\right)}{\partial \hat{\mu}_{X}^{m} \partial \hat{\mu}_{Y}^{n}}\right|_{\vec{\mu}=0},
$$

where $X, Y=B, Q, S, C$ and $\vec{\mu}=\left(\mu_{B}, \mu_{Q}, \mu_{S}, \mu_{C}\right)$ and $\chi_{0 n}^{X Y} \equiv \chi_{n}^{Y}$ and $\chi_{m 0}^{X Y} \equiv \chi_{m}^{X}$. In an uncorrelated gas of hadrons, i.e. described by the HRG model, the pressure of all the strange hadrons can be decomposed into mesonic part $P_{M}^{H R G}$ and baryonic part $P_{B}^{H R G}[4]$

$$
P_{S}^{H R G}\left(\mu_{B}, \hat{\mu}_{S}\right)=P_{|S|=1, M}^{H R G} \cosh \left(\hat{\mu}_{S}\right)+\sum_{\ell=1}^{3} P_{|S|=i, B}^{H R G} \cosh \left(\hat{\mu}_{B}-\ell \hat{\mu}_{S}\right) .
$$

Note that Boltzmann statistics is assumed here. For strange hadrons this is ensured since even the mass of the lightest strange hadron, i.e. Kaon, has the value of $\sim 3 T_{c}$.

Combinations of fluctuations, i.e. $v_{1}=\chi_{31}^{\mathrm{BS}}-\chi_{11}^{B S}$ and $\chi_{2}^{B}-\chi_{4}^{B}$ should vanish for an uncorrelated gas of hadrons within the classical Boltzmann approximation since the baryon-strangeness correlation differing by even number of $\mu_{B}$ derivatives are identical; $v_{2}=\frac{1}{3}\left(\chi_{2}^{S}-\chi_{4}^{S}\right)-2 \chi_{13}^{B S}-4 \chi_{22}^{B S}-2 \chi_{31}^{B S}$ denoting the differences of two operators each corresponding to the partial pressure of all strange hadrons, should also vanish in the uncorrelated hadron resonance gas. Here $v_{1}$ and $v_{2}$ receive contributions only from strange hadrons while $\chi_{2}^{B}-\chi_{4}^{B}$ receive contributions dominantly from light-quark hadrons. It is apparent from the left plot of Fig. 1 that all these three quantities deviate from the value of zero given by HRG almost at the same temperature, i.e. in the vicinity of chiral crossover temperature as indicated by the yellow band. This suggests that the strange degrees of freedom are liberated from hadrons at almost same temperature as light quarks. The whole analysis has been repeated using a different fermion action in Ref. [5].
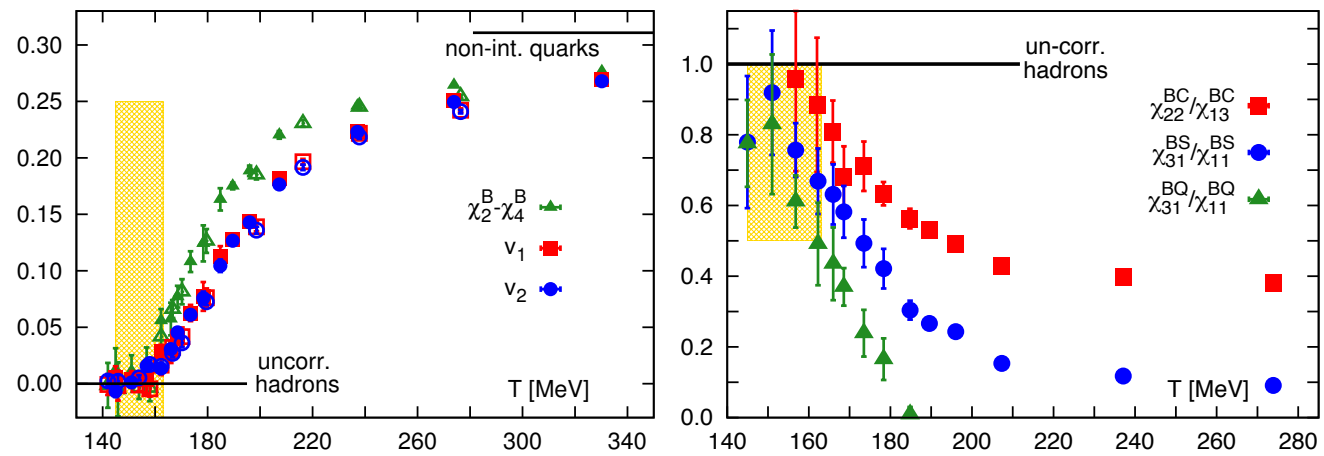

Figure 1. Breaking-down of uncorrelated hadron resonance gas description for charge correlations involving light, strange and charm quarks at temperatures in or above chiral crossover region as indicated by the yellow band. The left plot shows the charge correlations for light and strange sectors [4] while the right plot shows the results involving light, strange and charm quark sectors [6].

Based on the same idea one can also discuss the fate of charmed hadrons in the hot medium [6]. As in an uncorrelated hadron resonance gas the charmed baryon sector with charm quantum number $C= \pm 1$ dominates the contribution to the partial pressure of all charmed hadrons, $\chi_{n m}^{B C} \simeq \chi_{11}^{B C}$ with $n+m$ even and larger than 2. Thus three quantities, $\chi_{22}^{B C} / \chi_{13}^{B C}, \chi_{31}^{B S} / \chi_{11}^{B S}$ and $\chi_{31}^{B Q} / \chi_{11}^{B Q}$, which receive contributions only from charm, strange and light quark sectors, respectively, should be equal to unity within a description by an uncorrelated hadron resonance gas model. As can be clearly seen from the right plot of Fig. 1 that such a description breaks down for baryonic correlations involving light, strange, or charm quarks in or just above the chiral crossover region. 
The above study through the fluctuations of conserved quantum numbers give the information on the properties of open heavy hadrons at finite temperature. However, it is not able to tell anything on the fate of open heavy hadrons in specific channels and bound states with zero net charm and strange quantum number. To investigate the fate of various species of bound states one has to look into their spectral functions. The spectral functions $\rho_{H}(\omega, T)$ are related with the two-point Euclidean correlation functions as follows

$$
\begin{aligned}
G_{H}(\tau, T) & =\int_{0}^{\infty} \mathrm{d} \omega \frac{\cosh (\omega(\tau-1 / 2 T))}{\sinh (\omega / 2 T)} \rho_{H}(\omega, T), \\
G_{H}(z, T) & =\int_{0}^{\infty} \frac{2 \mathrm{~d} \omega}{\omega} \int_{-\infty}^{\infty} \mathrm{d} p_{z} e^{i p_{z} z} \rho_{H}\left(\omega, p_{z}, T\right) .
\end{aligned}
$$

Here $G_{H}(\tau, T)$ and $G_{H}(z, T)$ are temporal and spatial correlation functions in a hadronic channel $H$, respectively. As shown in the above equations the relation between spectral function and temporal correlation function is more straightforward, the spectral functions are thus commonly extracted from the temporal correlation functions rather than the spatial correlation functions. However, the extraction of spectral function are hampered by two issues: one is that the maximum temporal distance is restricted by the inverse temperature and the other one is the analytic continuation. The former one basically means that the physics in the temporal correlation functions are more compacted at higher temperatures and the latter tells that a large number of data points in the temporal direction is crucially required. Certain techniques, e.g. Maximum Entropy Method are normally used to extract the spectral functions [7].

The current state of art quenched lattice QCD study on charmonium spectral functions suggests that all charmonia are already dissociated at $1.5 T_{c}$ [8]. However, their dissociation temperatures have not yet been found. Recently an explorative lattice QCD study of charmonia properties in full QCD has been also made [9]. When moving to the case of heavier bound states, i.e. bottomonia, one can also perform lattice study based on an effective theory, i.e. Non-Relativistic QCD due to the large mass of bottom quark and the existence of certain hierarchies in the system. One pitfall of such study is that continuum limit is not available by definition, while the advantage is that the relation between temporal correlators and spectral functions becomes less complicated as the thermal boundary is removed. Similar results have been shown in recent studies on the fate of $\mathrm{S}$-wave and $\mathrm{P}$-wave bottomonia at finite temperature in both 2-flavour as well as 2+1-flavour QCD, i.e. $\mathrm{S}$ wave ground states survive up to at least $2 T_{c}$ while $\mathrm{P}$ wave ground states melt just above $T_{c}[10]$.
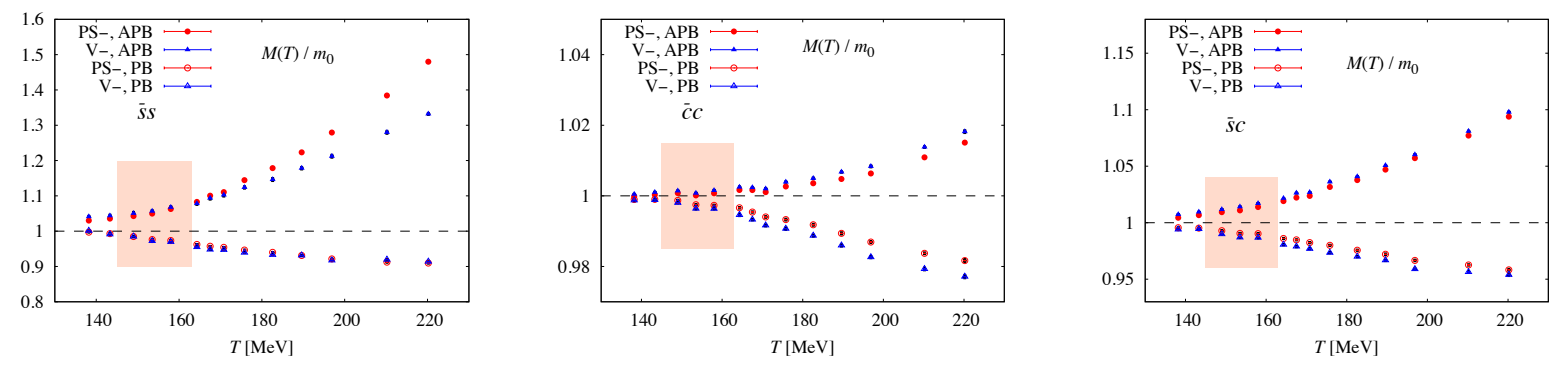

Figure 2. Ratios of screening masses at finite temperature to those at zero temperature for $s \bar{s}$ (left), $c \bar{c}$ (middle) and $\bar{s} c$ (right) systems. "PS-" stands for pseudo scalar channel while "V-" stands for vector channel. "APB" denotes anitiperiodic boundary condition and "PB" stands for periodic condition. Figures are taken from Ref. [11].

The modification of spectral function also manifests itself in the spatial correlation functions. As the physical extent in the spatial direction is not restricted by the temperature one can extract the exponentially decay constant, i.e. screening mass from the long distance behaviour of spatial correlators. At zero temperature a peak of low lying bound state with mass $m_{0}$ can be described by a spectral function $\rho\left(\omega, p_{z}, T\right) \sim \delta\left(\omega^{2}-p_{z}^{2}-m_{0}^{2}\right)$, thus the screening mass $M$ is the same as the pole mass $m_{0}$. In the high temperature limit a quark and its anti-quark are not bound anymore and the screening mass of the free quark pair is $2 \sqrt{(\pi T)^{2}+m_{q}^{2}}$, where $m_{q}$ is the quark mass and $\pi T$ is the lowest fermionic Matsubara frequency. The appearance of $\pi T$ in the free limit is due to the antiperiodicity (APB) of fermions along the time direction. Thus screening mass of a mesonic bound state is expected to be not sensitive in the boundary 
conditions, i.e. with periodic boundary conditions at $\mathrm{T}=0$ screening mass is still $m_{0}$ while in the free limit it becomes $2 m_{q}$. Changing the boundary conditions and comparing with the two temperature limit one can gain some information on the modification of bound states [12].

Full lattice QCD results on the ratio of screening mass to the pole mass for $s \bar{s}, c \bar{s}$ and $c \bar{c}$ states in both pseudo scalar and vector channels are shown in Fig.2[11]. It is apparent from the left plot of Fig. 2 that the bound states of $s \bar{s}$ suffer significant modifications already in the chiral crossover temperature region. This is consistent with the picture of unbounded open strange states in or above $T_{c}$ obtained from the study on the fluctuations of conserved quantum numbers seen in Fig. 1. For the $c \bar{c}$ states, only $\sim 2 \%$ deviation of $M$ from $m_{0}$ arises at $220 \mathrm{MeV}$, i.e. $\sim 1.4 T_{c}$. This plausible inconsistency with the quenched lattice QCD study on temporal and spatial correlation functions [8, 13] seems to stem from the effects of dynamic quarks, e.g. in the quenched limit the phase transition is first order while in the full QCD it is a rapid crossover. In the right plot of Fig. 2 results for open charm states $s \bar{c}$ are shown. The magnitude of deviation is larger than that of $c \bar{c}$ states. This further strengthen the point that open charm hadrons starts to melt at almost same temperature as light and open strange hadrons as seen from Fig. 1 .

Another recently developed approach to study the properties of heavy quarkonia states is based on the heavy quark effective theory. One of the main outcomes is that the static heavy quark potential has both imaginary and real parts [14]. These parts can be obtained through lattice QCD calculations on the correlators of Wilson lines. Serval studies have been performed along this interesting line [15, 16] and this topic was covered in much more details by A. Mócsy in this conference [17].

\section{Electromagnetic properties of QGP}

Dileptons/photons are good probes of QGP properties as they do not participate in the strong interaction and can carry the information of QGP produced in the early stage. However, they are produced almost during all the stages of heavy ion collisions. It is thus important to have a detailed knowledge of dilepton/photon rates radiated from QGP. The thermal dilepton/photon rates are related to vector spectral functions in the following way:

$$
\begin{aligned}
\frac{\mathrm{d} W_{l^{+} l^{-}}}{\mathrm{d} \omega \mathrm{d}^{3} \mathbf{p}} & =C_{e m} \frac{\alpha_{e m}^{2}}{6 \pi^{3}} \frac{1}{\omega^{2}\left(e^{\omega / T}-1\right)} \rho_{V}(\omega, \mathbf{p}, T), \\
\omega \frac{\mathrm{d} R_{\gamma}}{\mathrm{d}^{3} p} & =C_{e m} \frac{\alpha_{e m}}{4 \pi^{2}} \frac{\rho_{V}(\omega=|\mathbf{p}|, T)}{\mathrm{e}^{\omega / T}-1}
\end{aligned}
$$

where $\alpha_{e m}$ is the electromagnetic fine structure constant and $C_{e m}$ is the sum of the square of the elementary charges of the quark flavor $f, C_{e m}=\sum_{f} Q_{f}^{2}$. The emission rate of soft photons is also related to electrical conductivity $\sigma$ as follows

$$
\lim _{\omega \rightarrow 0} \omega \frac{\mathrm{d} R_{\gamma}}{\mathrm{d}^{3} p}=\frac{3}{2 \pi^{2}} \sigma(T) T \alpha_{e m},
$$

where $\sigma(T)$ is defined as the limiting behaviour of spectral function

$$
\frac{\sigma}{T}=\frac{C_{e m}}{6} \lim _{\omega \rightarrow 0} \lim _{\mathbf{p} \rightarrow 0} \frac{\rho_{i i}(\omega, \mathbf{p}, T)}{\omega T}
$$

Continuum extrapolated quenched lattice QCD results on thermal spectral functions and their resulting thermal dilepton rates as well as electrical conductivity are shown in Fig. 3 . In the high frequency region, i.e. $\omega / T \gtrsim 7$, all the thermal spectral functions at 1.1, 1.2 and $1.4 T_{c}$ can be well described by the Born rate as well as the Hard Thermal Loop (HTL) calculations. In the lower frequency region it is obvious that the lattice QCD results differ from the Born rate and HTL calculations. The negligible temperature dependence of thermal dilepton rate and $\sigma(T) / T$ as shown in the middle and right plots of Fig. 3 may due to the fact that the phase transition in the pure glue system is just a first order and at $T>T_{c}$ the temperature is thus the only relevant scale in the system.

The lattice QCD studies on electrical conductivities have been extended to full QCD recently [19]. The temperature dependence of electrical conductivity from recent calculations in both systems with and without dynamics quarks are summarised in the left plot of Fig. 4 [19-21]. At $T>T_{c}$ there is an obvious increasing of $\sigma(T) / T$ with temperature. This is different from the case in the pure glue system as shown in the right plot of Fig. 3 . Below $T_{c}$ 

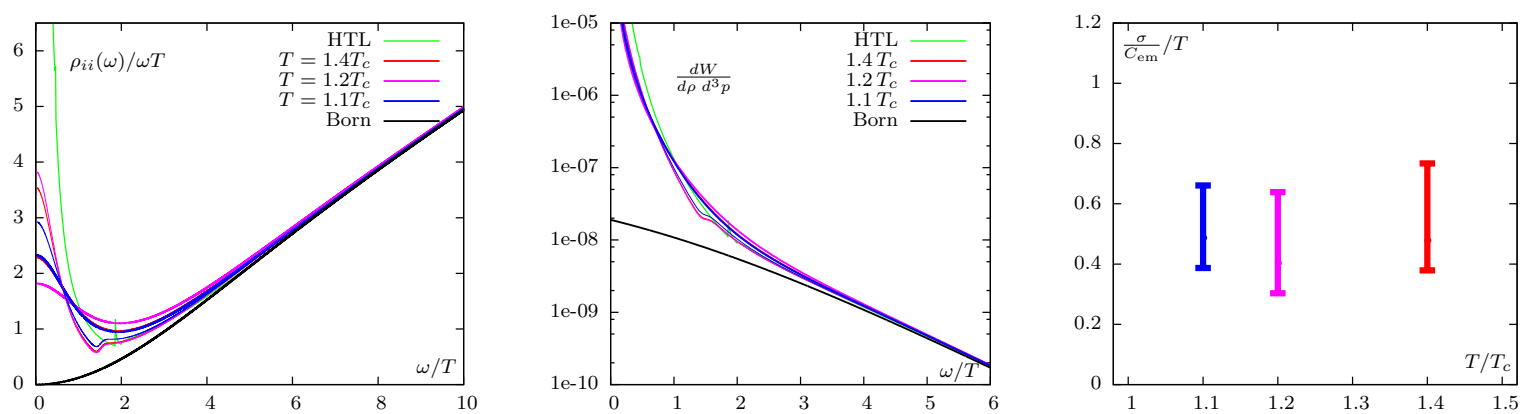

Figure 3. Temperature dependences of thermal spectral functions (left), thermal dilepton rates (middle) and electrical conductivities (right) at three different temperatures above $T_{c}$ obtained from quenched lattice QCD calculations [18].

$\sigma(T) / T$ seems to first remain as constant and then drop down to nearly zero at around $0.6 T_{c}$. The observed vanishing of electrical conductivity at $0.6 T_{c}$ might be due to the insensitivity of the temporal correlators to the low frequency behaviour of the spectral function. Besides the peak of $\rho$ meson with unphysical width at $0.63 T_{c}$ as seen from the right plot of Fig. 4 may also make the extraction of electrical conductivity more unreliable. Nevertheless it might be interesting to have a look at the so-called charge diffusion coefficient, i.e. $\sigma$ divided by light quark number susceptibilities, and have a comparison with the heavy quark diffusion coefficient as they probably have similar temperature dependence. However, to get a quantitative determination of electrical conductivities of a QCD system with dynamical quarks further lattice studies are still needed. This is due to fact that the main difficulty in practice to extract spectral functions from temporal correlators is the number of points in the temporal direction $\left(N_{\tau}\right)$. And obviously there is no reason that uncertainties of results obtained from temporal correlators with smaller $N_{\tau}$, e.g. in Ref. [19], should be smaller than those shown in Ref. [20]. The systematic uncertainties from analytic continuation also need to be examined using different methods[22].
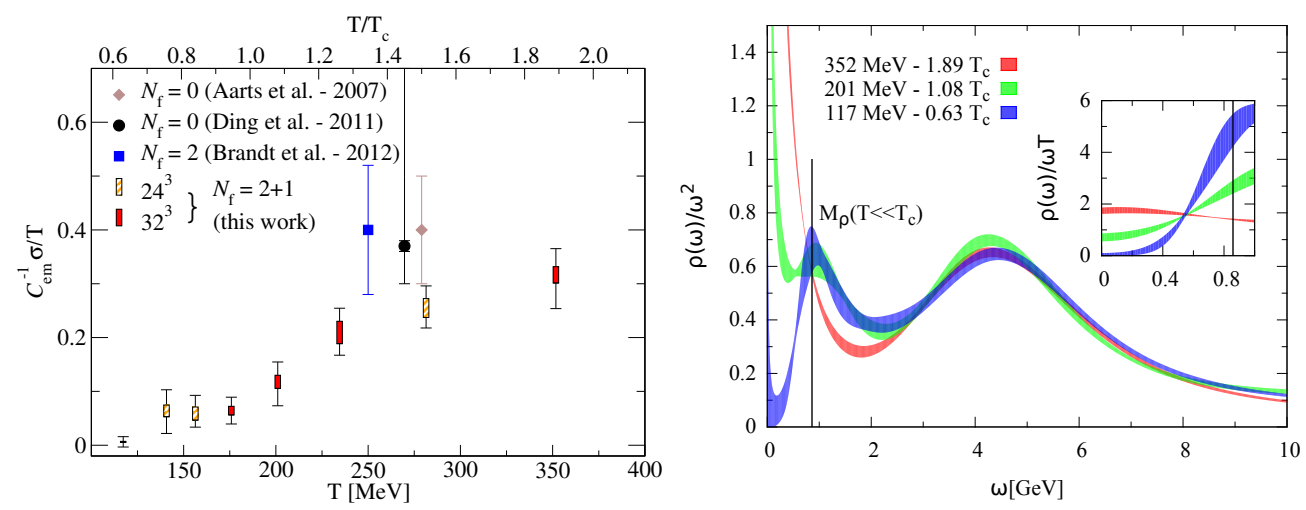

Figure 4. Temperature dependences of electrical conductivities (left) and spectral functions at three temperatures (right). Figures are taken from Ref. [19].

\section{Transport properties of QGP}

The Brownian motion of a heavy quark in the hot medium can be described by Langevin equation whose crucial input parameter is the heavy quark diffusion coefficient $D$. Based on the linear response theory, this dynamical quantity can be expressed as the low frequency limit of the vector spectral function obtained from Euclidean twopoint correlation functions. The temperature dependence of charm quark diffusion coefficient $D_{c}$ has been studied on 
the quenched lattice. It is obtained that $D_{c}$ is compatible with zero at $T<T_{c}$ and $2 \pi T D_{c}$ is independent of temperature and is around 2 in the temperature region from $\sim 1.5 T_{c}$ to $\sim 3 T_{c}$ [8]. These results are much smaller than those obtained from pQCD calculations [23].

Instead of extracting heavy quark diffusion coefficient from a meson spectral function as in Ref. [8], one can make use of Heavy Quark Effective Theory to define the diffusion coefficient through the low frequency limit of the spectral function related with a certain Euclidean correlation function involving color-electric fields along a Polyakov loop [24]. The usual inversion problem to obtain spectral functions from correlators still exists in this approach, however, the advantage is that the spectral function at low frequency is expected to be flat and does not receive contributions from hadron states appearing in meson spectral functions. Consistent estimates on $D T$ have been obtained by two different groups [25, 26] and these results are 2 3 times larger the charm quark diffusion coefficient obtained in Ref. [8]. Systematic uncertainties as well as continuum limit are certainly needed in the further study on diffusion coefficients in both studies based on QCD and HQEFT.

Jet quenching parameter $\hat{q}$, which is generally used to quantify the energy loss and multiple scatterings of energetic partons traversing in the medium, is another important transport coefficient of the medium. The difficulty to evaluate this quantity in lattice QCD is simply that $\hat{q}$ is defined along the light cone with real Minkowski time $t$. Several proposals have been made recently to calculate $\hat{q}$ on the lattice [27,-29]. Among them the attempt to evaluate $\hat{q}$ on the lattice using a purely Euclidean and dimensionally reduced effective theory, i.e. electrostatic QCD (EQCD), is based on the fact that the differential collision rate of partons with the medium from soft modes is almost time independent [27]. Preliminary results at two temperatures, i.e. $\sim 2 T_{c}$ and $\sim 10 T_{c}$ have been obtained [30]. At these temperatures contributions from ultrasoft modes might be small as results from EQCD can describe the lattice data of second and fourth order of quark number susceptibilities reasonably well at temperatures above $2 T_{c}$ [31]. However, further studies, e.g. with better improved action and finer lattice spacings are still needed.

\section{References}

[1] A. Bazavov, T. Bhattacharya, M. Cheng, C. DeTar, H. T. Ding, S. Gottlieb, R. Gupta and P. Hegde et al., Phys. Rev. D 85, 054503 (2012).

[2] S. Borsanyi et al. [Wuppertal-Budapest Collaboration], JHEP 1009(2010) 073.

[3] T. Matsui and H. Satz, Phys. Lett. B 178 (1986) 416.

[4] A. Bazavov, H. -T. Ding, P. Hegde et al., Phys. Rev. Lett. 111 (2013) 082301.

[5] R. Bellwied, S. Borsanyi, Z. Fodor, S. D. Katz and C. Ratti, Phys. Rev. Lett. 111 (2013) 202302.

[6] A. Bazavov, H. -T. Ding, P. Hegde, O. Kaczmarek, F. Karsch, E. Laermann, Y. Maezawa and S. Mukherjee et al., arXiv:1404.4043 [hep-lat].

[7] M. Asakawa, T. Hatsuda and Y. Nakahara, Prog. Part. Nucl. Phys. 46 (2001) 459.

[8] H. -T. Ding, A. Francis, O. Kaczmarek, F. Karsch, H. Satz and W. Soeldner, Phys. Rev. D 86 (2012) 014509.

[9] S. Borsanyi, S. Durr, Z. Fodor, C. Hoelbling, S. D. Katz, S. Krieg, S. Mages and D. Nogradi et al., arXiv:1401.5940 [hep-lat].

[10] G. Aarts et al., arXiv:1402.6210 [hep-lat], JHEP 1312 (2013) 064, JHEP 1111 (2011) 103.

[11] Y. Maezawa, A. Bazavov, F. Karsch, P. Petreczky and S. Mukherjee, arXiv:1312.4375 [hep-lat].

[12] F. Karsch, E. Laermann, S. Mukherjee and P. Petreczky, Phys. Rev. D 85 (2012) 114501, M. Cheng et al., Eur. Phys. J. C 71 (2011) 1564.

[13] H. Ohno, arXiv:1311.4565 [hep-lat].

[14] M. Laine, O. Philipsen, P. Romatschke and M. Tassler, JHEP 0703 (2007) 054.

[15] Y. Burnier and A. Rothkopf, Phys. Rev. Lett. 111 (2013) 182003.

[16] A. Bazavov, Y. Burnier and P. Petreczky, arXiv:1404.4267][hep-lat].

[17] A. Mócsy, this proceedings.

[18] O. Kaczmarek and M. Müller, arXiv:1312.5609 [hep-lat], M. Müller et al., PoS ConfinementX (2012) 185.

[19] A. Amato, G. Aarts, C. Allton, P. Giudice, S. Hands and J. -I. Skullerud, Phys. Rev. Lett. 111 (2013) 172001.

[20] H. -T. Ding, A. Francis, O. Kaczmarek, F. Karsch, E. Laermann and W. Soeldner, Phys. Rev. D 83 (2011) 034504.

[21] B. B. Brandt, A. Francis, H. B. Meyer and H. Wittig, JHEP 1303 (2013) 100.

[22] Y. Burnier and M. Laine, Eur. Phys. J. C 72 (2012) 1902.

[23] S. Caron-Huot and G. D. Moore, Phys. Rev. Lett. 100 (2008) 052301

[24] S. Caron-Huot, M. Laine and G. D. Moore, JHEP 0904 (2009) 053.

[25] A. Francis et al., arXiv:1311.3759 [hep-lat], A. Francis et al., PoS LATTICE 2011 (2011) 202.

[26] D. Banerjee, S. Datta, R. Gavai and P. Majumdar, Phys. Rev. D 85 (2012) 014510,

[27] S. Caron-Huot, Phys. Rev. D 79 (2009) 065039, M. Laine and A. Rothkopf, arXiv:1310.2413 [hep-ph].

[28] A. Majumder, Phys. Rev. C 87 (2013) 3, 034905.

[29] X. Ji, Phys. Rev. Lett. 110 (2013) 26, 262002.

[30] M. Panero, K. Rummukainen and A. Schäfer, arXiv:1307.5850 [hep-ph], M. Panero, K. Rummukainen and A. Schäfer, arXiv:1407.2233

[31] A. Bazavov, H. -T. Ding, P. Hegde, F. Karsch, C. Miao, S. Mukherjee, P. Petreczky and C. Schmidt et al., arXiv:1309.2317 [hep-lat]. 